\title{
Taking a spiritual history
}

\author{
Larry Culliford
}

Abstract The rationale for assessing the spiritual needs of psychiatric patients is examined as a prelude to addressing the question of how to do this. A number of approaches are considered. The emphasis is on varieties of practical history-taking suitable for the repertoire of every psychiatrist in training and those engaged in CPD. Finally, some of the consequences of identifying spiritual needs in psychiatric patients are discussed.

Spirituality links the deeply personal with the universal (Culliford, 2002b). It has a lot to do with individual subjective experience and, according to the researcher David Hay, it is rooted in human awareness (Hay \& Nye, 2006). On the basis of extensive studies involving both adults and children, Hay reports persuasively that, rather than being a social or cultural construction, 'It is really there' (p. 18). 'It' can be thought of as a spontaneous and consistently operating communicating principle, connecting individuals to one another via a seamless and indivisible whole. This entirety exists as a kind of sacred unity, and is referred to by some as 'creation'. The faculty of spiritual awareness appears better developed (or, according to David Hay, less completely atrophied) in some than in others.

Unlike religion, which tends to be associated with particular buildings, artefacts and scriptures, with rules and commandments, with trained officials, repetitive ceremonies and dogma, spirituality might be experienced as warmer and more spontaneous, associated rather with love, inspiration, wholeness, depth and mystery; with personal devotion and meditation, rather than with collective prayer and worship. A person's sense of spiritual connection is with humanity at large, rather than with exclusive or partisan groups.

Spirituality and religion are obviously vitally linked, and Hay uses metaphors to describe the dynamic interaction between them: spirituality as a journey with the religions as different modes of transport; spirituality as the fuel enabling the machinery of religion to operate; spirituality as the roots and trunk of a tree, of which the different religions are the branches and leaves. A spiritual history should include details of a person's religious antecedents (or lack of them), but this is only one component.

\section{Why take a spiritual history?}

A few years ago in the pages of APT I discussed the relevance of spirituality to mental healthcare (Culliford, 2002a). The reasons for taking a spiritual history in psychiatry are complex. The more obvious include:

- the very nature of spirituality as a source of vitality, motivation and a healthy sense of belonging and being valued

- the long historical relationship between religion, medicine and mental healthcare

- the patient's needs and wishes

- the epidemiology of spirituality/religion and mental health

- the influence of spirituality/religion on the attitudes and decisions of psychiatric staff.

Spirituality is universal, unique to every person. It is essentially unifying and involves everyone, including those who do not believe in God or a 'higher being'. Ellison has suggested that spirituality

'enables and motivates us to search for meaning and purpose in life. It is the spirit which synthesises the total personality and provides some sense of energising direction and order. The spiritual dimension does not exist in isolation from the psyche and the soma. It affects and is affected by our physical state, feelings, thoughts and relationships' (Ellison, 1983).

Spirituality is thus supraordinate to, and an integrating force for, the other hierarchically arranged dimensions of human life: physical, biological,

Larry Culliford is a consultant psychiatrist with the Sussex Partnership NHS Trust (Brighton Community Mental Health Centre, 79 Buckingham Road, Brighton BN1 3RJ, UK. Email: larry.culliford@sussexpartnership.nhs.uk) and a popular author as 'Patrick Whiteside' (see: http:// www.happinesssite.com). A practising Christian with wide ecumenical and inter-faith interests, he is a member of the Scientific and Medical Network (http:// www.scimednet.org) and the International Thomas Merton Society (http:/ /www.merton.org). 
psychological and psychosocial (Culliford, 2002a, 2007). Nevertheless, it is a dimension that has, until recently, been neglected in both physical and mental healthcare (Swinton, 2001). This neglect can largely be ascribed to the secularisation of the culture in which the mainly science-based discipline of psychiatry has developed.

Secularisation is a complex word that in Western culture initially referred to the divorce of personal spirituality from organised religion. This initial position then led to secularisation of the intellect, and in turn gave reason primacy over other major mental faculties: actions, sense perceptions, emotions and, particularly, intuition.

This division, and the resulting imbalance among these seamlessly and dynamically interrelated faculties, became more extreme and entrenched, partly in response to the perceived conflict between religion and science, for example in regard to evolutionary theory. In medicine and psychiatry, dualistic 'either/or' thinking continues to prevail over the more holistic 'both/and' style (Culliford, 2007). Renewed balance is called for.

Although opinion polls indicate that religious beliefs and practices are in decline, spirituality remains strong (Hay \& Hunt, 2000). The relevance of this for healthcare professionals and especially for psychiatric staff is that at times of emotional stress, illness, loss, bereavement and death people confront what Buckley (1987: p. 360) has called 'the great issues of life', that lie 'far beneath the formal separation of the sciences, and of the sciences from the humanities'.

In dealing with these weighty matters, mental health professionals can best help themselves, their colleagues in other disciplines and their patients by using ordinary language rather than religious terminology. In this way they will hit spontaneously on what Nolan \& Crawford (1997) call a 'rhetoric of spirituality'. Taking a spiritual history involves engaging people as equals in enquiry and discussion, using their own words, about what - at the deepest level - makes sense to them and what puzzles them, what motivates them and what holds them back. This is the most direct way to get quickly to the heart of whatever is troubling the patient. It coincides with the essence of good medical practice: two people, doctor and patient, engaged in genuine and meaningful communication about what matters most. It seems worth adding that both may gain from the encounter.

It is not surprising that, where spirituality is concerned, patients' needs and wishes coincide (Faulkner, 1997). As Greasley et al (2001) note, spirituality is a vital concern for most service users. In a study of spiritual care in mental health practice, Nathan (1997) asked psychiatric patients to describe
Box 1 Key elements of spiritual care from the patient's perspective

- An environment fostering hope, joy and creativity

- Being valued and trusted, treated with respect and dignity

- Sympathetic and confidential listening

- Help to make sense of, and derive meaning from, illness experiences

- Receiving permission, encouragement (and sometimes guidance) to develop spiritually

(Adapted from Nathan, 1997)

\section{Box 2 Benefits of spiritual care}

- Healthy grieving of losses (letting go)

- Improved self-esteem and confidence

- Maximisation of personal potential

- Improved relationships (with self, others and with the Absolute/God)

- Renewed sense of meaning and purpose

- Enhanced feeling of belonging

- Improved capacity for solving problems

- Insoluble problems, continuing distress and disability are more easily endured

- Hope renewed

(Adapted from Nathan, 1997)

the most important elements of 'spiritual care' and the potential benefits such care may bring. Their responses are summarised in Boxes 1 and 2.

Aspiritual history is most clearly necessary when spiritual or religious issues are part of the presenting problem, for example in religious delusions, feelings of rejection (by God or a faith group) and excessive guilt or shame. Although it is acknowledged that religion can have negative effects, confidence is growing in the benefits to both physical and mental health of spiritual beliefs and practices. This confidence is based on substantial epidemiological research of improving quality (Koenig et al, 2001; Levin, 2001). These authors suggest that, whereas $20 \%$ of studies report negative effects, $80 \%$ identify spiritual/religious beliefs and practices as beneficial, not so much part of the problem as part of the remedy (Box 3).

The influence of spirituality/religion on the attitudes and decisions of psychiatric staff is also complex. Although curricula exist (Puchalski \& Larson, 1998), in the UK the topic has seldom been taught as part of professional training as it needs to be: in terms of knowledge, skills and attitudes. When individual views on the subject have been 
Box 3 Positive effects of spirituality and religion on mental health

- In addictions (e.g. Alcoholics Anonymous and similar groups using the 12-step method)

- Enabling of inner resources (e.g. sources of hope and calm)

- Connecting or reconnecting with external resources (e.g. within the person's faith community)

canvassed, it is not surprising that mental healthcare professionals' attitudes towards spirituality have tended to be negative (Neelman \& King, 1993).

At one extreme, any expression of religiosity or spiritual awareness might automatically be deemed psychopathological. Routinely taking patients' spiritual histories, and becoming more skilful at doing so, will provide staff with both information and material for reflection. The experience and knowledge thus gained will help correct any previously held attitudinal bias.

At the other extreme, caring efficiently and compassionately for disadvantaged others can legitimately be experienced vocationally, as part of a sacred and undeniable calling. Many mental health workers consider themselves to some degree spiritually guided. For these, the taking of spiritual histories will be an expression of spiritual caregiving, and therefore fulfilling in itself, as well as being a necessary preparation for dealing appropriately with patients' spiritual needs. The benefits of assessing spirituality are therefore many and widespread.

\section{How to take a spiritual history}

Taking a spiritual history is best thought of as a clinical skill to acquire and hone, rather than as an activity to be performed by recipe or rote. It is a skill that requires empathic engagement with the patient, which therefore sanctions the judicious use of both intuition and initiative on the part of the assessor. This may lead, for example, to sensitive exploration of what the patient only hints at or seems to be avoiding. Such exploration is to honour and uphold the spiritual values of truth and truthfulness, the enemy of which is concealment, whether conscious or otherwise. It is important to seek to avoid witting or unwitting collusion with the patient. It is therefore wise always to be reasonably systematic and, thereby, thorough.

The interview works best in a comfortable, quiet and confidential setting, and a gentle, unhurried approach is recommended. More than one conversation may be necessary.

\section{Brief screening}

Notwithstanding the ideal of thoroughness, it is sometimes necessary to make rapid assessments of psychiatric patients. At such times, two main types of question are useful:

- 'Are you particularly religious or spiritual?'

- 'What helps you most when things are difficult, when times are hard?' (for example when facing big problems, major losses or important challenges).

The first question might lead the psychiatrist to ask the patient more directly whether they are atheist, agnostic, unsure, religious or 'spiritual but not religious' (Box 4).

The patient's reply to the second question usually points to the principal values they hold and to what is most meaningful in their life, and is indicative of their major spiritual concerns and practices (Box 5).

Supplementary non-directive questions may be necessary, for example 'Would you like to say more about that?'

\section{Box 4 Suggested definitions for spiritual} identities

Atheist To be atheist is emphatically to deny the existence (even the possibility) of a sacred being, supreme reality, god or other deity

Agnostic To be agnostic is to assert that we cannot truly know about the existence or otherwise of a sacred being, supreme reality, god or other deity

Unsure To be unsure is not to know what one believes about a sacred being, supreme reality, god or other deity

Religious To be religious is to believe and have faith in a sacred being, supreme reality, god or other deity, and/or to belong to and practice within an organised religion (people might see themselves as both religious and spiritual)

Spiritual To be spiritual (but not religious) is to believe and have faith in a sacred being, supreme reality, god or other deity, but to be independent and not belong to or practise within an organised religion 


\section{Box 5 Common spiritual practices}

Mainly religious

- Belonging to a faith tradition, participating in associated community-based activities

- Ritual and symbolic practices and other forms of worship

- Pilgrimage and retreat

- Meditation and prayer

- Reading scripture

- Sacred music (listening to, singing and playing), including songs, hymns, psalms and devotional chants

Mainly secular

- Acts of compassion, in everyday life and as part of one's work, especially teamwork

- Deep reflection (contemplation)

- Yoga, tai chi and similar disciplines

- Engaging with and enjoying nature

- Contemplative reading (of literature, poetry, philosophy, etc.)

- Appreciation of the arts and engaging in creative activities, including artistic pursuits, cookery, gardening, etc.

- Maintaining stable family relationships and friendships (especially those involving high levels of trust and intimacy)

An appropriate next step, even in a brief screening, would be to ask in more detail about spiritual practices. Regularly engaging in such activities identifies a person as spiritually engaged as much as does holding and expressing spiritual or religious beliefs. Listing one or more 'mainly secular' spiritual practices may give cause to people who describe themselves as atheist, agnostic or unsure to reconsider their selfassessment, and - whatever beliefs they have - to begin to accept themselves as in some way 'spiritual' and therefore spiritually influenced.

\section{Taking a more detailed spiritual history: five approaches}

A brief screening will often indicate that a more detailed history is required to establish relevant aspects of the patient's background, specific problems related to spirituality or religion, available spiritual supports and additional spiritual needs.

Various authorities have separately designed guidance on assessing the religious and spiritual aspects of people's lives. However, they are fairly uniform regarding the topics covered. This allows practitioners to pick the style with which they feel most comfortable. Guides tend to take the form of

\section{Box 6 Some types of spiritual experience}

- Mystical experiences (broadly defined)

- Near-death experiences

- Twelve-step spirituality (as in Alcoholics Anonymous and similar programmes)

- Dreams

- Psychedelic (drug-induced) states

an aides-memoire rather than exact prescriptions. Here I will mention only five of these.

Inthefirst, publishedbytheSpiritualCompetencyand Resource Centre (http: / / www.spiritualcompetency. com/assess_spirit/ASrshx.asp), questions focus on: religious background and beliefs; spiritual meaning and values (e.g. spiritual practices (Box 5) and spiritual experiences (Box 6)); and prayer experiences.

Second, in her guide to the assessment of spiritual concerns in mental healthcare, Eagger (2005) lists some simple, non-intrusive questions that can inform the care team's approach. These look, for example, at the place of spirituality or religion in the patient's past and present, the nature of its influence (positive or negative, supportive or excluding) and whether they affect the patient's acceptance of and engagement in treatment

Third, the leaflet Spirituality and Mental Health (Royal College of Psychiatrists, 2006) suggests five broad areas of questioning: setting the scene; the past; the present; the future; and remedies. These gather a picture of how patients see themselves, their place and purpose in life and their future, and ask whether spirituality or religion are part of the problem and could be part of the solution.

Fourth, in an article aimed at healthcare professionals in general, Puchalski \& Romer's (2000) guide to taking a spiritual history uses the mnemonic FICA:

- Faith and belief (what gives the patient's life meaning)

- Importance (how important this is to their situation)

- Community (their place in any social or religious group)

- Address in care (how they would like their beliefs to be addressed in their healthcare).

Finally, another tool for spiritual assessment is the HOPE questions (Anandarajah \& Hight, 2001). The mnemonic HOPE directs the assessor's attention to four areas of the patient's life:

- sources of Hope, meaning, comfort, strength, peace, love and connection 
- Organised religion

- Personal spirituality and Practices

- Effects on medical (psychiatric) care, and Endof-life issues.

\section{After taking a spiritual history, what next?}

\section{Formulation}

Formulation is the term used in psychiatry for summarising and making sense of information gathered and observations made while taking a history, examining the physical and mental state of the patient and conducting specific biophysical and psychological tests. Formulation is not a goal or endpoint. Its primary purpose is to assist the patient.

Formulation is a skilled process that helps clarify where an assessment is incomplete and therefore what useful information remains to be gathered. Most formulations are therefore provisional, and should be revised regularly as observations continue and information develops.

The simplest type is the diagnostic formulation. Once the diagnosis is reasonably established, short-term treatment plans can be devised and implemented. Depending on their outcome and other developments, medium- and long-term treatment plans will follow. Some refer to this diagnosistreatment approach as invoking the medical model of mental healthcare.

A more comprehensive, and therefore preferable, approach involves the bio-psychosocial type of formulation, in which problems and their solutions are sought under headings involving three dimensions of human experience. This is a big step towards holism, towards considering the symptoms and problems in the context of the whole person, and the person in the context of family, community and culture. Including a spiritual history allows this process to be completed, through what may be referred to as a bio-psychosocio-spiritual (BPSS) formulation (the terms psychospiritual, personcentred and holistic are also used).

The BPSS formulation still has as its primary purpose the well-being of the patient, acting as a guide to clinicians' planning and execution of helpful interventions.

\section{How may a spiritual approach contribute to patient welfare?}

\section{Better rapport}

Clinicians regularly discover that taking a spiritual history - enquiring attentively about patients' primary concerns and motivating factors - deepens rapport and improves its quality. Feeling valued as individuals, patients often relax and invest further trust in the doctor, thus improving the therapeutic alliance.

\section{Clarifying psychotic symptoms}

There are two main ways in which taking a spiritual history can help clarify psychotic symptoms.

First, it helps distinguish 'spiritual emergence' from psychosis. Originally called 'spiritual emergency', this refers to a destabilising period of rapid spiritual growth (Vega, 1989; Grof, 2000; Slade, 2004).

Second, existential questions such as 'What is the purpose of my life?' are conundrums that become problematic for many. Religious grandiosity of delusional strength may be a powerful but immature defence against meaningless insignificance. As part of a psychotic reaction, such symptoms may be common to those feeling particularly unworthy or unloved, and their religious content hints at a spiritual solution.

To be psychotic and consider oneself divine is satisfying only narcissistically, through a false inner sense of supremacy. It does get people's attention, however, in a way that, often accompanied by corresponding feelings of persecution, may foster the individual's sense of grandiosity. It is better that such a person, when well enough, is encouraged to understand the true origins of their distress and work towards more modest, mature and acceptable ways of gaining meaning, recognition and satisfaction in daily life. This mirrors the cognitive-behavioural therapy approach but with an extra dimension. Only what may be called 'spiritual sustenance' will be effective against the degree of insignificance and all-consuming meaninglessness that can be at the heart of psychotic and other disorders. Pastoral or spiritual support and spiritual practices may both appropriately be recommended in such cases.

\section{'Psychoneuroses'}

Anxiety and depression are key elements in a range of non-psychotic psychiatric disorders, particularly the 'psychoneuroses'. Another universally experienced existential problem derives from emotional attachments. As soon as these are formed, they render people vulnerable to the threat of loss and to loss itself.

Anxiety, bewilderment and doubt are emotions associated with the threat of loss. Anger, the emotion of resistance, arises as loss becomes more likely and imminent. Depressive emotions - shame, guilt and sadness - emerge when a loss increasingly becomes an acknowledged reality (Culliford, 2007). 


\section{Box 7 Useful websites and webpages}

- Spiritual Competency Resource Center

- Royal College of Psychiatrists' Spirituality and Psychiatry Special Interest Group

- George Washington Institute for Spirituality and Health

- American Academy of Family Physicians: Anandarajah \& Hight's (2001) paper on using the HOPE Questions

- Alcoholics Anonymous

- Narcotics Anonymous

- Gamblers Anonymous

- University of Minnesota's Center for Spirituality and Healing (online learning about spirituality in healthcare and free module on taking a spiritual history) http://www.spiritualcompetency.com/index.asp

http://www.rcpsych.ac.uk/spirit

http://www.gwish.org/index.htm

http:/ / www.aafp.org/afp/20010101/81.html

http://www.alcoholics-anonymous.org.uk

http://www.ukna.org

http://www.gamblersanonymous.org.uk

http://www.csh.umn.edu/modules/index.html
Taking a spiritual history involves enquiry about a person's primary attachments, whether to a spiritual reality, to family and other loved ones, to places and objects, or to ideas and ideals. Identifying the major attachments, and the spectrum of emotions arising in response to threatened and actual loss, encourages emotional flow towards acceptance and resolution. Taking a spiritual history is therefore intrinsically therapeutic. It helps to clarify for the patient that these emotions are normal and healthy, part of their pathway to psychological growth and maturity through the acceptance of losses and resolution of the emotional healing process (Culliford, 2007). This reflects another principle of spirituality, that personal growth results more often through facing and enduring adversity, rather than from trying to avoid it.

In some cases of severe anxiety and / or depression, there is a profound sense of meaninglessness and personal insignificance, as described above. Spiritual advice and support may again appropriately be recommended, and spiritual practices helpful.

\section{Addictions}

The value of a spiritual approach is specifically acknowledged by those who advocate or follow the twelve-step method of dealing with addiction. The best known organisations to use this approach are Alcoholics Anonymous (where it originated), Narcotics Anonymous and Gamblers Anonymous. Narcotics Anonymous, for example, describes itself as 'a non-religious fellowship, encouraging each member to cultivate an individual understanding, religious or not, of a spiritual awakening' ('What is NA?', http://www.ukna.org).
The heart of a programme of personal recovery is contained in twelve steps that describe the experience of the earliest members of Alcoholics Anonymous (http://www.alcoholics-anonymous. org.uk/geninfo/05steps.shtml). ${ }^{1}$ Newcomers are not required to accept or follow the twelve steps in their entirety if they feel unwilling or unable to do so. The key step for addicts is to recognise and respect some form of spiritual reality, manifest particularly as a higher power: 'Soon we came to believe in a power greater than ourselves' (http:// www.gamblersanonymous.org.uk/young.htm).

\section{Others disorders}

Psychiatrists see a number of other conditions that might have a spiritual element in their aetiology. Absence or removal of meaning and sense of purpose affect drive and motivation. Having a damaged sense of belonging affects self-esteem and a person's true and healthy sense of identity. These elements may occur, for instance, in personality disorder, eating disorder and chronic fatigue syndrome, as well as in disorders already mentioned here. There may be persistent psychological resistance to loss, in the form of intense anger, often denied and either repressed or more consciously suppressed. Enquiry into these central and vital aspects of a person's life is part of spiritual history-taking, and it offers an important opportunity to reframe the problem in terms that may lead to reintegration and healing.

\footnotetext{
1. The twelve steps of Alcoholics Anonymous have been published in a previous issue of APT: see Luty, J. (2006) What works in alcohol use disorders? Advances in Psychiatric Treatment, 12, 13-22. Ed.
} 


\section{Involving chaplaincy services}

Sensitive enquiry is in itself helpful for patients whose needs and problems have a spiritual dimension, and it may allow them with greater clarity and efficacy to fulfil their own needs and find their own solutions. However, they may seek spiritual support and guidance, and therefore be in need of pastoral care. Referral to a chaplain or pastoral care advisor is often appropriate. Such a referral is necessary if more complex issues such as spirit possession are involved.

\section{Chaplaincy}

After psychiatric staff have taken a spiritual history from a patient, a well-informed and experienced chaplain, prepared to see and assess the patient, should be available for consultation and advice. ${ }^{+}$ Mental healthcare providers and trusts should maintain a multi-faith chaplaincy service with adequate staffing levels. Voluntary part-time as well as paid full-or part-time chaplains and pastoral care staff will be required.

Although many chaplains and spiritual advisors will be involved only in general and supportive work, some are increasingly valued as contributors to the work of multi-disciplinary mental health services. If they work in that capacity they should receive appropriate training in mental health matters. In return, chaplains will probably have made a point of establishing good relations with local clergy and faith communities, and will provide a knowledge base about local religious groups, their traditions and practices. They will be alert to situations in which religious beliefs and activities may prove harmful to individuals or groups, and suitably trained chaplains will also be available for advice on controversial issues such as spirit possession and the ministry of deliverance.

\section{Conclusions}

\section{According to the Australian writer David Tacey,}

'Enlightened people everywhere live according to the light of reason and logos, but we all also need a mythos, a spiritual belonging, to make life meaningful and bearable. Mythos provides a goal, offers dignity, and establishes a relationship to past, present and future' (Tacey, 2006)

${ }^{+}$Collaboration between psychiatric and religious professionals has been discussed briefly in an earlier APT article: Dein, S. (2004) Working with patients with religious beliefs. Advances in Psychiatric Treatment, 10, 287-294. Ed.
In answer to Hay's question then, what is 'really there' is a spiritual dimension of human experience that provides the context for everything else. For those with any measure of spiritual awareness, this is both the source and the goal of existence. To take systematic and detailed spiritual histories regularly will enable practitioners to rekindle mythos in medicine and put the psyche back into psychiatry. The psyche, of course, is our soul.

\section{Declaration of interest}

L.C. is on the Executive Committee of the Royal College of Psychiatrists' Spirituality and Psychiatry Special Interest Group.

\section{References}

Anandarajah, G. \& Hight, E. (2001) Spirituality and Medical Practice: Using the HOPE Questions as a Practical Tool for Spiritual Assessment. American Family Physician, 63, 8192

Buckley, M. (1987) At the Origins of Modern Atheism. Yale University Press.

Culliford, L. (2002a) Spiritual care and psychiatric treatment: an introduction. Advances in Psychiatric Treatment, 8, 249-258.

Culliford, L. (2002b) Spirituality and clinical care. BMJ, 325, 1434-1435.

Culliford, L. (2007) Love, Healing and Happiness: Spiritual Wisdom for Secular Times. O Books.

Eagger, S. (2005) A Short Guide to the Assessment of Spiritual Concerns in Mental Healthcare. Royal College of Psychiatrists. http:/ / www.rcpsych.ac.uk/PDF/DrSEaggeGuide.pdf

Ellison, C. W. (1983) Spiritual well-being: conceptualization and measurement. Journal of Psychology and Theology, 11, 4.

Faulkner, A. (1997) Knowing Our Own Minds: A Survey of How People in Emotional Distress Take Control of Their Lives. Mental Health Foundation

Greasley, P., Chiu, L. F., \& Gartland, Revd M. (2001) The concept of spiritual care in mental health nursing. Journal of Advanced Nursing, 33, 629-637.

Grof, S. (2000) Psychology of the Future: Lessons from Modern Consciousness Research. SUNY Press.

Hartz, G. (2005) Spirituality and Mental Health: Clinical Applications. Haworth Press.

Hay, D. \& Hunt, K. (2000) Understanding the Spirituality of People Who Don't Go to Church: A Report on the Findings of the Adults' Spirituality Project. University of Nottingham.

Hay, D. \& Nye, R. (2006) The Spirit of the Child (revised edn). Jessica Kingsley.

Koenig, H. K., McCullough, M. E. \& Larson, D. B. (2001) Handbook of Religion and Health. Oxford University Press.

Levin, J. (2001) God, Faith and Health. John Wiley \& Sons.

Nathan, M. M. (1997) A Study of Spiritual Care in Mental Health Practice: Patients' and Nurses' Perceptions. MScThesis. Middlesex University.

Neelman, J. \& King, M. (1993) Psychiatrists' religious attitudes in relation to their clinical practice: a survey of 231 psychiatrists. Acta Psychiatrica Scandinavica, 88, 420-424.

Nolan, P. \& Crawford, P. (1997) Towards a rhetoric of spirituality in mental health care. Journal of Advanced Nursing, 26, 289294.

Puchalski, C. \& Larson, D. (1998) Developing curricula in spirituality and medicine. Academic Medicine, 73, 970-974.

Puchalski, C. \& Romer, A. (2000) Taking a spiritual history allows clinicians to understand patients more fully. Journal of Palliative Medicine, 3, 129-137.

Royal College of Psychiatrists (2006) Spirituality and Mental Health. Royal College of Psychiatrists. http:/www. 
rcpsych.ac.uk/mentalhealthinformation/therapies/ spiritualityandmentalhealth.aspx

Slade, N. (2004) Heavenbound. In Spirituality and Mental Health: Breakthrough (eds P. Barker \& P. Buchanan-Barker), pp. 167190. Whurr.

Swinton, J. (2001) Spirituality and Mental Health Care: Rediscovering a Forgotten Dimension. Jessica Kingsley.

Tacey, D. (2006) Spirituality and the future of health. Journal of the Scientific and Medical Network, 91, 7 -10.

Vega, S. (1989) Spiritual Emergence or Psychosis? http://www. spiritmoving.com/papers/SpiritPsychosis.htm

World Health Organization (1998) WHOQOL and Spirituality, Religiousness and Personal Beliefs: Report on WHO Consultation. WHO.

\section{MCQs}

\section{Spirituality:}

a is something that applies only to a few special people

b comes to the fore at times of emotional stress, loss and the threat of loss

c bears limited relationship to a person's physical state, feelings, thoughts and relationships

d usually divides people and is a source of conflict

e depends on holding strong religious convictions.

2 Taking a spiritual history:

a serves no useful purpose

b does not require empathic engagement

c concentrates on a person's beliefs

d should only be undertaken by chaplains or ministers of religion

e can be therapeutic for the patient.

\section{Spiritual practices:}

a are mainly religious activities

b do not include everyday activities such as gardening or walking in the country

c tend to heighten a person's spiritual awareness over time

d require repetition and ritual to be effective

e can only be undertaken in groups.

4 Practical ways of taking a spiritual history include:

a the HOPE questions

b the Minnesota Multiphasic Personality Inventory

c the Royal Free Interview for religious and spiritual beliefs

d the Mini-Mental State Examination

e The twelve-step method.

5 A psychospiritual assessment may help patients by:

a resulting in a miraculous and instantaneous cure of symptoms

b encouraging them to pray regularly

c identifying specific spiritual practices that they should undertake regularly in addition or as an alternative to prayer

d leading to an appropriate referral for pastoral care and spiritual support

e encouraging passive acceptance in the face of divine will.

\section{MCQ answers}

$\begin{array}{lllllll}\text { 1 } & & \text { 2 } & & 3 & 4 & 5 \\ \text { a F } & \text { a F } & \text { a F } & \text { a T } & \text { a F } \\ \text { b T } & \text { b F } & \text { b F } & \text { b F } & \text { b F } \\ \text { c F } & \text { c F } & \text { c T } & \text { c F } & \text { c F } \\ \text { d F } & \text { d F } & \text { d F } & \text { d F } & \text { d T } \\ \text { e F } & \text { e T } & \text { e F } & \text { e F } & \text { e F }\end{array}$

